\title{
SOME CONSIDERATIONS ON AN UNDERWATER ROBOTIC MANIPULATOR SUBJECTED TO THE ENVIRONMENTAL DISTURBANCES CAUSED BY WATER CURRENT
}

\author{
Waldemar KOLODZIEJCZYK ${ }^{*}$ \\ *Faculty of Mechanical Engineering, Department of Automatic Control and Robotics, Bialystok University of Technology, \\ ul. Wiejska 45c, 15-351 Bialystok, Poland \\ w.kolodziejczyk@pb.edu.pl
}

received 31 July 2015, revised 19 February 2016, accepted 22 February 2016

\begin{abstract}
The objective of this paper is to discuss some of the issues associated with environmental load on the three-link serial manipulator caused by underwater current. We have conducted CFD simulations to investigate hydrodynamic effects induced by changing current direction and changing with time current speed in order to better understand the physics of the problem. The results are presented in terms of moments of hydrodynamic forces plotted against relative position of the current and the robotic arm. Time history of hydrodynamic loads according to periodically changing current speed is presented and discussed.
\end{abstract}

Key words: Underwater Manipulator, CFD, Current Disturbances

\section{INTRODUCTION}

Underwater manipulators constitute subsystems of remotely operated vehicles (ROVs) or autonomous underwater vehicles (AUVs), making the jobs of exploration, construction, inspection, and recovery possible. Manipulators enable the robots to replicate the function of human arm in carrying out operations such as picking up objects from the bed, placing and joining assembly parts, drilling, cutting, however, the overall performance of underwater manipulation is greatly affected by interaction with the water environment, particularly in position/motion control of the endeffector with respect to the given target position.

Land-based manipulators operate in the air, which is much lighter than a solid body, and consequently, their models of $d y-$ namics, usually, do not include environmental contributions, as though they move in vacuum, not meeting any resistance at all. In underwater applications the impact of the environment cannot be neglected, and in consequence, quantifying of hydrodynamic loads is necessary, especially, in determining controllability of the system. Moreover, the underwater robotic arms have to be designed to withstand the hydrodynamic forces exerted by the surrounding fluid.

Underwater manipulators consist of rigid links connected in series by pin joints. The joint torques for generating the motion of the links have to overcome the weight and payload along with the hydrodynamic load induced by relative motion of the arm and the fluid. A moving solid body immersed in flowing water is subjected to the pressure and shear stress distributions resulting from their relative motion. In the equations of motion of the multibody system this hydrodynamic load is usually decoupled into independently computed inertia, friction damping and lift contributions, although these phenomena are strongly coupled according to basic continuum mechanics principles.

Currents represent important ambient flow conditions in a changeable and uncertain aquatic environment. Ocean cur- rents are caused by tidal movements, wind, heat exchange, the salinity differences, and the Coriolis force due to the rotation of the Earth. Furthermore, the refresh of water in structured environment may be strong enough to induce additional hydrodynamic loads on the underwater system. Usually the current effect on the underwater manipulator is modeled (if at all) in a simplified way as a constant disturbance by assuming the current to be irrotational and unchanged (Fossen, 1994; Antonelli, 2006). Real ocean currents are often multi-directional and irregular, spatially and time variable.

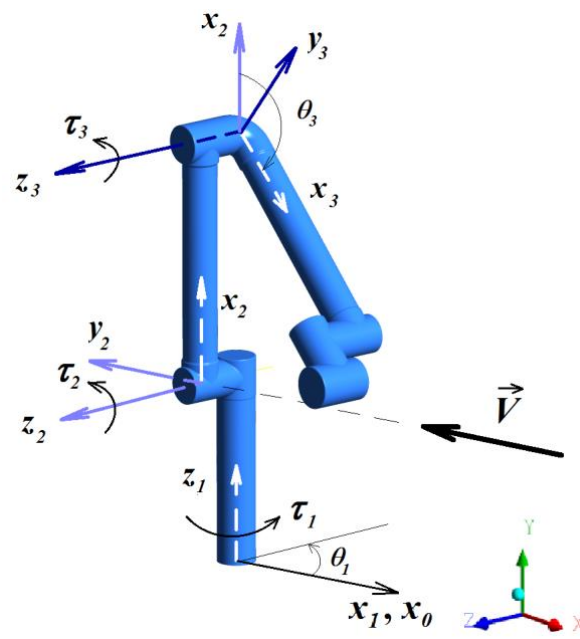

Fig. 1. Coordinate frame arrangement of the robotic arm (external and local reference frames)

In this paper some aspects of hydrodynamic load on the underwater robotic arm caused by current of water are considered and analyzed by using the CFD modeling tools in order to get more insight to the physics of the problem. The power of CFD 
simulations enabled us to observe the effects of disturbances such as changes in the direction of the current velocity and its magnitude on the moments experienced by the manipulator joints.

This paper is organized as follows. The second section is devoted to the modeling of water environmental impact on the manipulator considered as a lumped mechanical system. In the third section the continuum mechanics approach and CFD modeling of water flow around the robotic arm is described. The next section provides results of calculations and discussion, and the last section - conclusions.

\section{MECHANICAL ASPECTS}

The main purpose to model a robot manipulator is to compute the forces and torques required to drive the joints given the requested end-effector position. The other purpose is to simulate the motion of the robotic arm in order to define control strategies. The hydrodynamic effects have to be assessed in advance to reproduce in a proper way the dynamical behavior of the robotic arm, otherwise the control may be significantly deteriorated. Recently, it is increasingly performed in specialized virtual prototyping environments, utilizing lumped approximations to hydrodynamic forces. Some of these environments were described in Wang (2012).
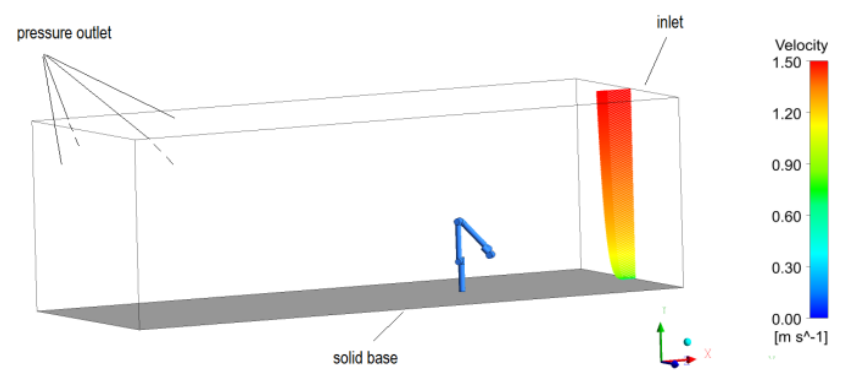

Fig. 2. Manipulator, computational domain, types of boundary conditions and velocity profile at the inlet to the domain

The robotic arm considered in this study is a serial manipulator (Fig. 1). It is composed of three links with diameters of $8.4 \mathrm{~cm}$. The lowest link is $0.43 \mathrm{~m}$ long, the middle one $-0.45 \mathrm{~m}$, and the upper link has the cylindrical part of the length of $0.4 \mathrm{~m}$. The configuration of the arm was kept unchanged at the position of the third upper link inclined at an angle $\theta_{3}=135^{\circ}$ with respect to the middle vertical link (Fig. 1).

Industrial or, more generally, land-based manipulators operate in the air, and consequently their equations of motion do not have to include environmental contributions. Hydrodynamics matters in underwater applications. In the lumped modeling of the dynamics of the rigid body in water environment the additional inertia resistance due to the accelerated fluid is taken into account through the concept of added mass and added moments of inertia, depending on the shape of the body and its ability to carry along the fluid (Fossen, 1994; Antonelli, 2006).

The lumped model of dynamics of an underwater manipulator, considered as a rigid multibody system consists of equations of motion derived by using law of conservation of momentum. In case of underwater applications it is usually written in the following form (Antonelli, 2006; Herman, 2009; Pazmino et al., 2011):
$M \dot{\mathrm{v}}+C(\mathrm{v}) \mathrm{v}+D(\mathrm{v}) \mathrm{v}+g(\mathrm{v})=\tau_{\mathrm{v}}$,

where: $\mathrm{v}$ - is the velocity vector. The symbols:

$M=M_{m}+M_{a}, \quad C(\mathrm{v})=C_{m}(\mathrm{v})+C_{a}(\mathrm{v})$

denote as follows: $M_{m}$ and $C_{m}(\mathrm{v})$ are the rigid body mass matrix, and the Coriolis/centripetal matrix, respectively, whilst Ma denotes the added mass matrix and $\mathrm{C}_{\mathrm{a}}(\mathrm{v})$ - the added Coriolis/centripetal matrix. $D(v)$ stands for the drag matrix, $g(v)$ - is the resultant vector of gravity and buoyancy; $\tau_{\mathrm{v}}-$ is the resultant input vector of forces and moments influencing the manipulator.

A number of works have been conducted in order to model underwater manipulator dynamics. Richard and Levesque (1996) developed the dynamic model of subsea manipulator for a nondeterministic fluid environment in order to deal with stochastic buoyancy effects for partially immersed arm. McLain and Rock (1998) and Leabourne and Rock (1998) have estimated added mass and drag contributions using and developing potential flow theory and experimental tests. Vossoughi et al. (2004) investigated the dynamics of underwater robotic arm incorporating results obtained by McLain and Rock (1998) for modeling hydrodynamic effects in order to design velocity control system. Santhakumar and Kim (2012) developed indirect adaptive control system for Underwater Vehicle Manipulator System (UVMS) in order to compensate disturbances caused, inter alia, by currents.

The added terms are computed as the extra forces and moments, needed to accelerate the fluid, divided by the accelerations - linear or angular, respectively. They are usually expressed in terms of hydrodynamic coefficients.

The part of hydrodynamic force on the body, dependent on the relative velocity is usually decomposed into an in-line force, called the drag force, and a transverse force - the lift force. They are expressed in terms of hydrodynamic coefficients as well. Drag coefficients depending on the shape and configuration of the manipulator, and the type of the flow characterized by Reynolds number, are computed as a superposition of a constant part representing laminar flow and a velocity-depending part accounting for turbulent effects.

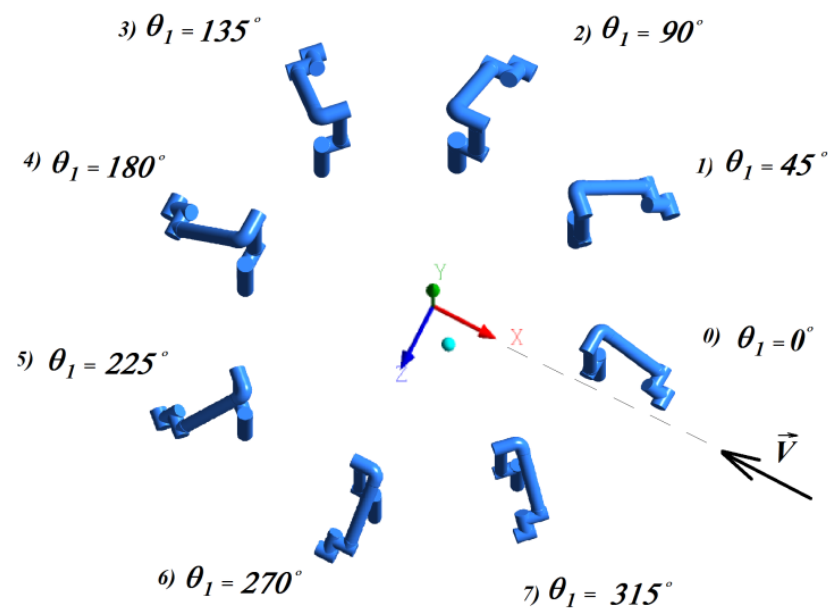

Fig. 3. The relative positions of the robotic arm and current velocity $\vec{V}$

The hydrodynamic added inertia and drag coefficients are commonly computed from the strip theory coming from potential flow background for $2 \mathrm{D}$ inviscid flows, and extended semiempirically to three dimensions (McLain and Rock, 1998). In this 
approach, the solid body is divided into multiple narrow slices, which can be considered as airfoils. Viscous effect of the fluid has to be incorporated into a model via additional drag and lift coefficients.

Water currents are usually assumed to be irrotational and constant and their effects are modeled as constant disturbances included into added inertia, added Coriolis/centripetal and damping terms of the model (Fossen, 1994; Antonelli, 2006).

The equations of motion are highly nonlinear due to hydrodynamic forces (Fossen, 1994). They contain uncertainties associated with hydrodynamic coefficients, estimated only approximately from simplified theories, experimental studies and empirical relations.

\section{HYDRODYNAMIC MODELING}

In the lumped approach, presented in the previous section, the hydrodynamic effect on the body completely immersed in water is decomposed into forces and moments depending on acceleration and velocity in relative motion between water and manipulator, which results in added inertia, added Coriolis/centripetal and damping contributions, taken into considerations independently, although in real flows these flow phenomena are strongly coupled, according to the fundamental conservation laws of continuum mechanics. In continuum mechanics approach all hydrodynamic forces and moments acting on the submerged manipulator are the result of pressure and shear stress distributions around the body, occurring in the flow.

Furthermore, in solid body mechanics the effect of the underwater current on the submerged body is commonly considered as being constant and irrotational, but real flow even far away from boundary layer on the body is always rotational and turbulent. This is another premise to employ the continuum mechanics approach and numerical simulations in the present considerations.

Tab. 1. Grid independence study

\begin{tabular}{|c|c|c|c|c|c|c|c|}
\hline \multirow{2}{*}{$\begin{array}{c}\text { SI. No. } \\
i\end{array}$} & \multirow{2}{*}{$\begin{array}{c}\text { Number } \\
\text { of cells } \\
N\end{array}$} & \multicolumn{3}{|c|}{ Torques [N m] } & \multicolumn{3}{|c|}{ Grid independence factor } \\
\hline & & $\tau_{1}$ & $\tau_{2}$ & $\tau_{3}$ & $\delta_{1}$ & $\delta_{2}$ & $\delta_{3}$ \\
\hline 1 & 2369004 & -3.56 & 5.31 & -9.88 & 0.177 & 0.341 & 0.182 \\
\hline 2 & 4242883 & -4.92 & 4.54 & -11.15 & 0.139 & 0.148 & 0.077 \\
\hline 3 & 7986142 & -4.44 & 4.05 & -12.50 & 0.027 & 0.025 & 0.023 \\
\hline 4 & 9747773 & -4.32 & 3.96 & -12.09 & & & \\
\hline
\end{tabular}

a)

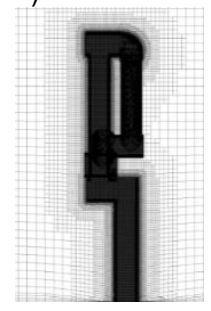

b)

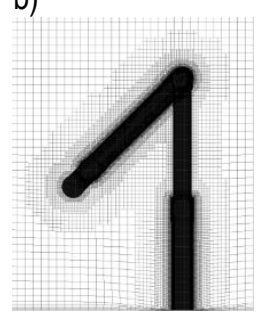

c)

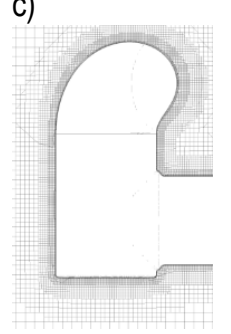

Fig. 4. Details of computational grid near and on the surface of the manipulator

In this research emphasis was placed on the calculations of moments of hydrodynamic forces exerted by the current of water about three $z$ axis in local reference frames assigned to the arm links, as they are shown in Fig. 1. Going from top to bottom, the moment $\tau_{3}$ was calculated taking into account pressure and shear stress distributions along the surface of upper link about $z_{3}$ axis. The moment $\tau_{2}$ includes hydrodynamic effects (due to pressure and shear stresses) on the two upper links with respect to $z_{2}$ axis and the moment $\tau_{1}$ - describes the action of water on the whole manipulator about $z_{1}$ axis. They can be considered as joint torques experienced by the manipulator placed into the current of water and which have to be compensated by motors in order to maintain the positions of the links.

Incompressible fluid flow surrounding the robotic arm, when temperature effect is neglected, is governed by the set of continuity equation and Navier-Stokes equation of motion.

For 3D problems, the governing equations can be described as:

- the continuity equation:

$\nabla \cdot \vec{V}=0$

- the Navier-Stokes equation of motion:

$\frac{\partial \vec{V}}{\partial t}+(\vec{V} \cdot \nabla) \vec{V}=-\frac{1}{\rho} \nabla p+\frac{\mu}{\rho} \nabla^{2} \vec{V}+f$,

where: $\vec{V}=\left[V_{x}, V_{y}, V_{z}\right]$ is the velocity vector, $\rho$ is the density of fluid, $p$ is the pressure, $\mu$ - the viscosity, $f-$ the body force (in this case - apparent weight) per unit mass, and $\nabla$ is the vector differential operator (gradient).

The present considerations were bounded to the computational domain in the shape of a box of $8 \mathrm{~m}$ long, $3 \mathrm{~m}$ wide and $2.5 \mathrm{~m}$ tall, depicted in Fig. 2. The manipulator was attached to the solid base in the middle of the width of the base at a distance of $2.5 \mathrm{~m}$ from free current inlet, as it is shown in Fig. 2. The other sides of computational domain were in contact with surrounding flowing water with the assumption that the backflow into the domain was allowed and computed using the direction of the flow in the cell layer adjacent to these sides (pressure outlet boundary conditions).

Gulf Stream, Kuroshio, Agulhas, Brazil, and East Australian Currents flow at speeds up to $2.5 \mathrm{~m} / \mathrm{s}$. It was decided to limit the velocity to $1.5 \mathrm{~m} / \mathrm{s}$ at most. This was the maximum current speed far away from the base in free stream of water. Reynolds number computed with respect to the links diameters and the maximum current speed was equal to 126000 . The $1 / 7$ th power law was used to specify turbulent velocity profile near the solid base at the inlet to the domain (see Fig. 2).

The considerations were carried out for two cases investigating two different current disturbances. The first one concerned the change in the direction of the current with respect to the position of the robotic arm. The second one examined the properties of the flow, when the speed of the current changes in time. The investigations of the current direction effect on the load on the manipulator were performed as steady-state, whilst the second ones, dealing with the time dependence of the current speed, were considered as transient problem. The change in the direction of the current with respect to the fixed robotic arm was modeled as the change in position of the arm (its rotation about vertical axis) to the unchanged current direction, because it was the easiest way to maintain the shape of the computational domain unchanged. The relative positions of the robotic arm and the direction of the current are indicated in Fig. 3 as the angles of rotation $\theta 1$ about vertical axis with respect to the initial position, when the third upper link is inclined exactly towards the current $\left(\theta_{1}=0\right)$. 
Tab. 2. Resolving moments into components due to viscosity $\tau_{\mathrm{v}}$ and pressure $\tau_{p}$

\begin{tabular}{|c|c|c|c|c|c|c|c|c|c|}
\hline & $\theta_{1}$ & $0^{\circ}$ & $45^{\circ}$ & $90^{\circ}$ & $135^{\circ}$ & $180^{\circ}$ & $225^{\circ}$ & $270^{\circ}$ & $315^{\circ}$ \\
\hline \multirow{3}{*}{$\begin{array}{c}\tau_{1} \\
{[\mathrm{Nm}]}\end{array}$} & $\tau_{\mathrm{v}}$ & -0.0497 & 0.0996 & 0.1724 & 0.1833 & 0.0483 & -0.0877 & -0.1379 & -0.1854 \\
\hline & $\tau_{p}$ & -4.2703 & 0.5536 & 4.2854 & 5.5586 & 1.0702 & -3.0283 & -5.4013 & -5.4408 \\
\hline & $\tau_{\mathrm{v}} / \tau_{p}$ & 0.012 & 0.179 & 0.040 & 0.033 & 0.045 & 0.029 & -0.026 & 0.034 \\
\hline \multirow{3}{*}{$\begin{array}{c}\tau_{2} \\
{[\mathrm{Nm}]}\end{array}$} & $\tau_{\mathrm{v}}$ & 0.407 & 0.2793 & 0.00276 & -0.3426 & -0.4648 & -0.3354 & 0.0229 & 0.3255 \\
\hline & $\tau_{p}$ & 3.548 & 3.3682 & -0.4384 & -6.0219 & -8.8238 & -7.4930 & -0.0560 & 3.4445 \\
\hline & $\tau_{\mathrm{v}} / \tau_{p}$ & 0.115 & 0.083 & -0.006 & 0.057 & 0.053 & 0.045 & -0.409 & 0.094 \\
\hline \multirow{3}{*}{$\begin{array}{c}\tau_{3} \\
{[\mathrm{Nm}]}\end{array}$} & $\tau_{\mathrm{v}}$ & -0.215 & -0.1721 & -0.00012 & 0.1555 & 0.1849 & 0.1511 & 0.0038 & -0.1492 \\
\hline & $\tau_{p}$ & -11.872 & -10.2525 & 0.04047 & 4.2664 & 5.1017 & 3.7452 & -0.1965 & -7.6076 \\
\hline & $\tau_{\mathrm{v}} / \tau_{p}$ & 0.018 & 0.017 & -0.003 & 0.036 & 0.036 & 0.040 & -0.019 & 0.019 \\
\hline
\end{tabular}

The ANSYS CFD (ANSYS, Canonsburg, PA) software was used to conduct simulations. For the computational domain with different manipulator positions to the current the set of eight meshes of approx. $950000 \div 10500000$ elements were generated using cut-cell method. Simulations were executed in Parallel Fluent 16.0 (which implements the finite volume method) with twelve parallel processes utilizing a second order spatial pressure discretization and second order upwind discretization schemes for momentum equations and for the model of turbulence. In steadystate simulations the SIMPLE algorithm (Semi-Implicit Method for Pressure Linked Equations) was used as the method of solution, whilst in transient simulation - the PISO (Pressure Implicit with Splitting of Operators) scheme was applied with second order implicit transient formulation and 20 iterations per time step equal to $0.01 \mathrm{~s}$.

Grid independence analysis was carried out for steady-state flow and initial arm position, i.e. for the rotation angle $\theta_{1}=0$, comparing resulting torques $\tau_{1}, \tau_{2}, \tau_{3}$, obtained for meshes of different resolutions, as it is shown in Tab. 1. Grid independence factor was defined as

$\delta_{j}=\left|\frac{\tau_{j}(i)-\tau_{j}(4)}{\tau_{j}(4)}\right|$,

where: $j$ is an indicator of the torque $(1,2$ or 3$), i$ - stands for a serial number of the mesh (Tab. 1), $\tau_{j}(4)$ is the " $j$ " torque computed for the reference grid No. 4 of maximum number of cells. As one can see in Tab. 1, grid independence factor constantly decreases with increasing number of cells and for two finest meshes of cell numbers 7986142 and 9747773 , the relative differences of the torques were less than $3 \%$. In order to better capture the flow structures, the finest mesh (No. 4) was chosen and, consequently, the number of cells for all computational cases was kept in the range of $950000 \div 10500000$. Fig. 4 depicts some details of the computational grid near and on the surface of the manipulator.

\section{RESULTS AND DISCUSSION}

A number of steady-state numerical simulations were conducted under a series of various environmental disturbances caused by different current directions. The influence of changing with time current speed has been studied utilizing transient simu- lations for the initial position of the arm $\left(\theta_{1}=0^{\circ}\right)$. The effects of these environmental disturbances on hydrodynamic load on the robotic arm are analyzed in the following subsections.

\subsection{The effect of current direction change}

Several numerical calculations were carried out for eight different relative positions of the arm and the current direction indicated by the angle $\theta_{1}\left(0^{\circ}, 45^{\circ}, 90^{\circ}, 135^{\circ}, 180^{\circ}, 225^{\circ}, 270^{\circ}\right.$, $315^{\circ}$ ). The angle $\theta_{1}$ is measured from the $\mathrm{x} 0$ axis directed opposite the flow as it is depicted in Fig. 1. The results are gathered and presented in Fig. 5 in terms of hydrodynamic torques $\tau_{1}, \tau_{2}$, $\tau_{3}$ plotted against the angle $\theta_{1}$. All the moments were calculated in local reference frames shown in Fig. 1.

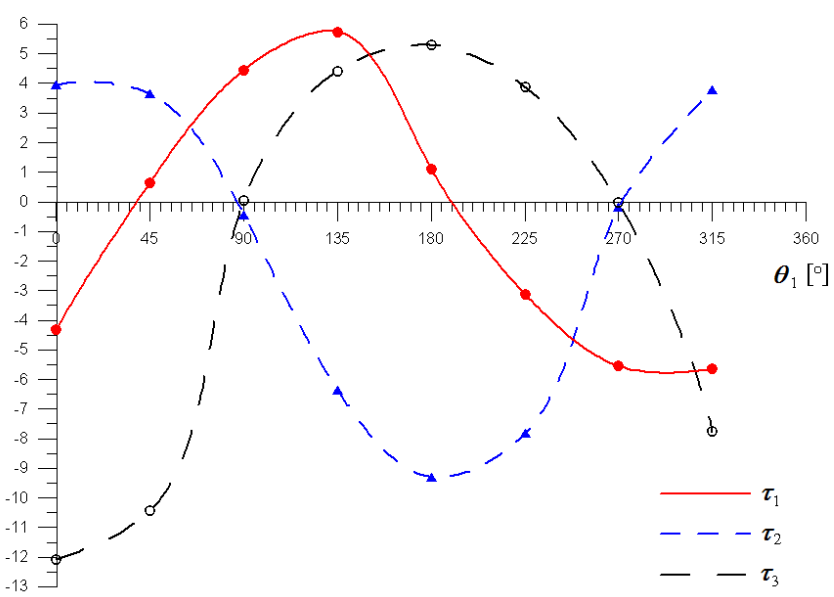

Fig. 5. Hydrodynamic torques vs. the angle $\theta_{1}$

The torque $\tau_{1}$ of the whole manipulator around vertical $z 1$ axis vanishes changing its direction at $\theta_{1}=38^{\circ}$ and $190^{\circ}$ mainly due to dissymmetry of the arm. It is a common practice for underwater vehicles and manipulator systems (UVMS) to align them with the current in order to minimize the drag forces, but in our case the torque $\tau_{1}$ at the position aligned with the flow $\left(\theta_{1}=0^{\circ}\right)$ was only a little less than maximum absolute value achieved. Taking into account, that drag forces are usually estimated only approximately, neglecting some geometric details of the system disturbing the 
symmetry of the structure, one has to be aware of inaccuracies induced by this practice. Even that dissymmetry of the structure, as we have in the case of our manipulator located at the initial position, can cause unanticipated increase of the joint torque required to maintain the position of the arm.

The maximum magnitude of $\tau_{1}$ was obtained for $\theta_{1}=135^{\circ}$ and between $270^{\circ} \div 315^{\circ}$. It is worth mentioning, that $\tau_{1}$ takes unexpectedly different values at corresponding positions with respect to the current, e.g. at $\theta_{1}$ equal to $135^{\circ}$ and $225^{\circ}$ or $45^{\circ}$ and $315^{\circ}$. The behavior of $\tau_{2}$ and $\tau_{3}$ is more predictable and intuitive. They are equal to zero for $\theta_{1}=90^{\circ}$ and $270^{\circ}$. Their plots are almost symmetrical with respect to $\theta_{1}=180^{\circ}$ and they take approximately the same values for corresponding positions.

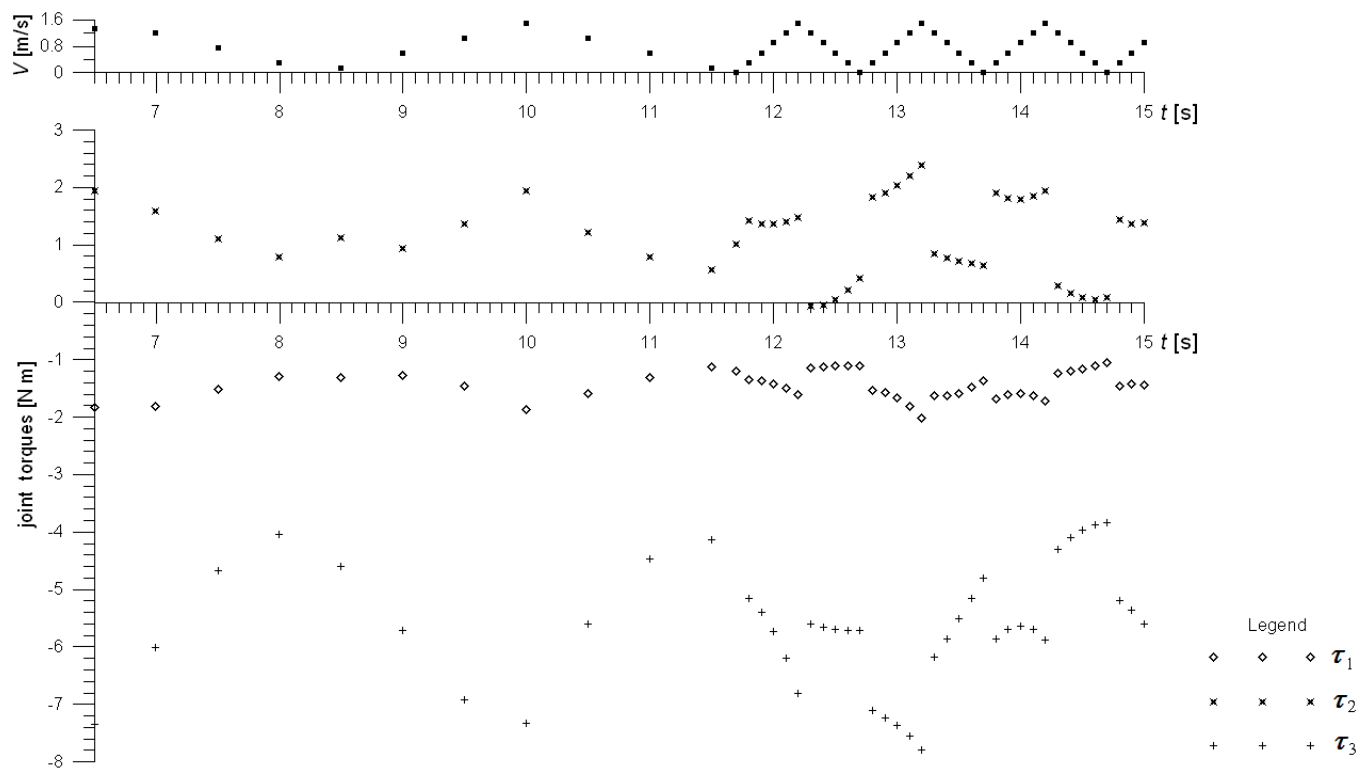

Fig. 6. Time history of joint torques $\tau_{1}, \tau_{2}, \tau_{3}$

Moments of hydrodynamic forces appear mainly due to the pressure distribution around the links. Tab. 2 shows moments $\tau_{1}$, $\tau_{2}, \tau_{3}$ broken into components $\tau_{\mathrm{v}}$ and $\tau_{\mathrm{p}}$, generated due to shear and pressure forces, respectively. Maximum magnitude of viscous $\tau_{\mathrm{v}}$ equal to about $0.5 \mathrm{Nm}$ acted on the two upper links with respect to $z_{2}$ axis (Fig. 1) at position of the arm indicated by $\theta_{1}=180^{\circ}$ (Fig. 3), but in most cases they were less than $0.1 \mathrm{Nm}$ and made significant fraction of pressure induced moments $\left(\tau_{v} / \tau_{p}\right)$ only when the pressure component notably decreases due to flow conditions (for example $\tau_{1}$ at the position indicated by $\left.\theta_{1}=45^{\circ}\right)$.

\subsection{The effect of changing with time current speed}

The effect of current speed was examined applying transient CFD simulation for time dependent inlet velocity to the domain. At the inlet to the computational domain turbulent velocity profile has been changing with time according to the saw function shown at the top of Fig. 6 . For the sake of clarity only a part of time history of joint torques $\tau_{1}, \tau_{2}, \tau_{3}$ is displayed, although simulation was performed over time duration of 17s and above, starting from initial conditions obtained from steady-state calculations for arm initial position denoted in Fig. 3 as $\theta_{1}=0$. The free stream speed has been changing linearly from zero to $1.5 \mathrm{~m} / \mathrm{s}$ there and back again with two different periods of change. In the first interval (below 11.7s) the period of speed change was equal to $3.33 \mathrm{~s}$, and after that it has been reduced to $1.0 \mathrm{~s}$.

As one can see from plots in Fig. 6 , the torques $\tau_{1}, \tau_{2}, \tau_{3}$ follow the changes of inlet velocity. It is especially clearly seen for the first time interval (below 11.7s), when speed frequency was fairly low. After reducing the time period to $1.0 \mathrm{~s}$, the behavior of the plots is not so obvious, but it can be observed, that the magnitudes of torques are increasing with increasing velocity and, in most cases, decreasing with decreasing speed, but changes become more irregular and violent. Velocity distributions depicted in Fig. 7 for time instances 14.2s and 14.7s, that is, when current speed sharply starts dropping or rising, respectively, show how agitated becomes the flow after increasing the frequency of inlet velocity changes (upstream the manipulator) compared with that of lower frequency (downstream the arm).

a)

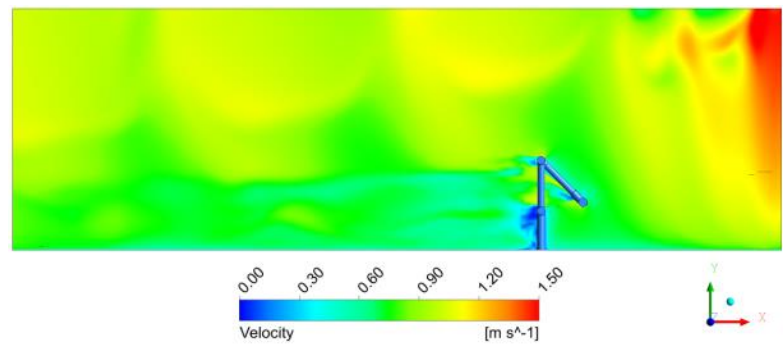

b)

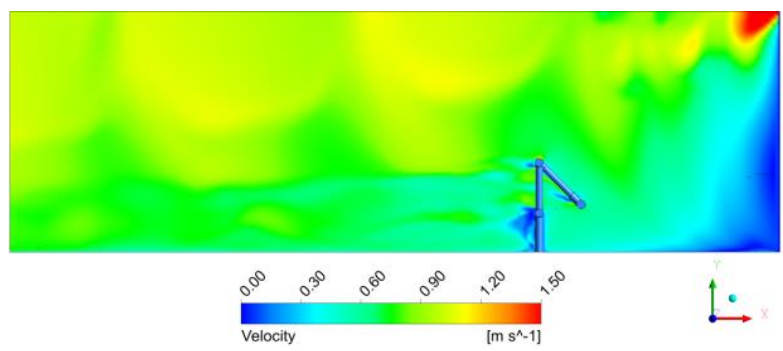

Fig. 7. Velocity distributions in the midplane of the domain of solution: a) $t=14.2 \mathrm{~s}$; b) $t=14.7 \mathrm{~s}$ 
In the second interval (beyond 11.7s), when the time period was very short (1.0s), sharp and sudden changes of torques magnitudes can be observed for sharp changes of speed, e.g. in $t=14.2 \mathrm{~s}$ and $14.7 \mathrm{~s}$. For lower frequency at an earlier time (below 11.7s), such phenomena were not discovered. The hydrodynamic loads expressed in terms of joint torques changed gradually with speed changes, much smoother than for higher speed frequency.
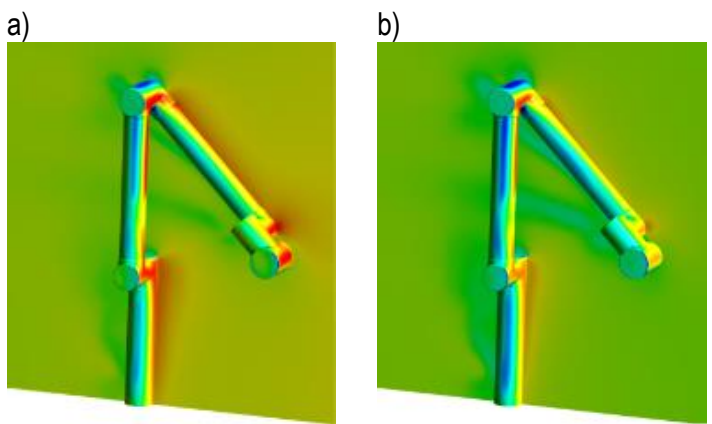

c)

d)
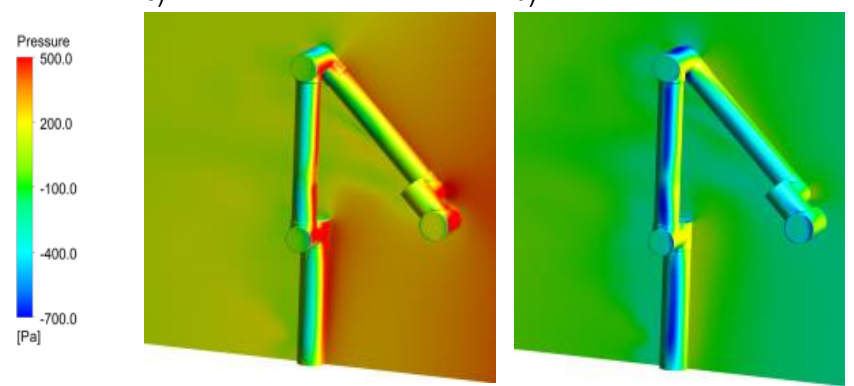

e) f)
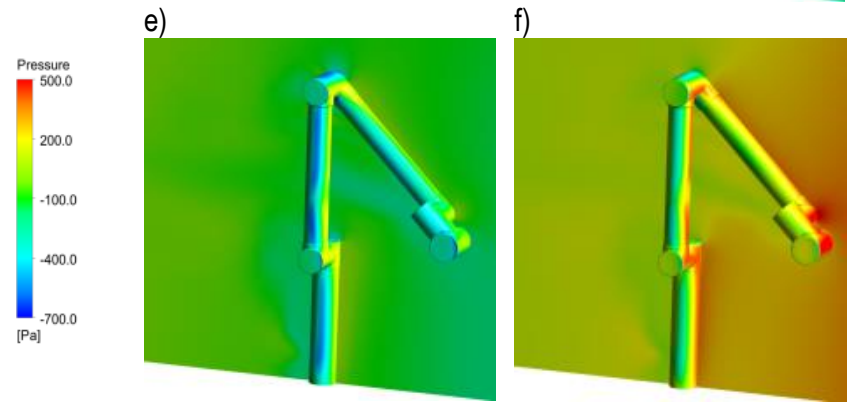

Fig. 8. Pressure (gauge pressure) distributions on the robotic arm and in the midplane of the domain in selected time points: a) $t=10.0 \mathrm{~s} ; \mathrm{b}) t=10.1 \mathrm{~s}$; c) $t=14.2 \mathrm{~s}$; d) $t=14.3 \mathrm{~s}$; e) $t=14.7 \mathrm{~s}$; f) $t=14.8 \mathrm{~s}$

The explanation to that flow behavior comes from pressure distribution analysis carried out on the basis of plots shown in Fig. 8. Suffice it to compare the pressure distributions on and in the vicinity of the manipulator, drawn for two subsequent time points, when the speed starts dropping for higher and lower frequency. For example, the pressure maps presented in Fig. 8c) and $8 \mathrm{~d}$ ), drawn for time $t=14.2 \mathrm{~s}$ and $t=14.3 \mathrm{~s}$, respectively, reveal much more intense changes in pressure than in corresponding situation for lower frequency (compare Fig. 8a and $8 b)$. The same conditions, but in opposite direction of pressure change, are when current speed starts rising, e.g. from the time point $t=14.7 \mathrm{~s}$ to $t=14.8 \mathrm{~s}$, for which pressure distributions are presented in Fig. 8e) and 8f), respectively. More intense pressure changes caused by greater acceleration of water for higher frequency induce sharp and sudden changes in joint torques depict- ed in Fig. 7. The fast variations in velocity always cause great variations in pressure in the surroundings, which cannot be reduced by the viscous damping of water. This type of flow behavior may happen due to sudden changes in flow velocity during, for example, the gate opening or closing in structured underwater environment.

It should be added at the end of this section, that in the kind of time dependent flow presented in this paper, periodically decreasing velocity causes the great reduction of the magnitude of hydrodynamic moments experienced by the manipulator. For example, the greatest magnitude of $\tau_{1}$ exceeds $12 \mathrm{Nm}$ in steady flow, whilst in time dependent flow it takes the values not more than $8 \mathrm{Nm}$.

The results obtained for changing current direction are more general than those for changing current speed. The latter ones depend strongly on the geometry of the computational domain, and especially on the distance of the manipulator from the source of disturbances (inlet), however they may serve as an example showing qualitatively the impact of such changeable environmental conditions as current speed.

\section{CONCLUSIONS}

In this paper attention is given to the physics of the problem of disturbances influencing underwater robotic arm, caused by varying current direction and speed. Realistic predictions of environmental loads are needed to identify operational limitations and set proper operating ranges while doing a task. Usually they are assessed applying simplified theories and experimental studies. Compared to experiments, CFD software offers a less expensive alternative, and can serve as a useful and very promising tool to solve this problem. There are several works carried out in the area of CFD modeling of underwater vehicles performed in order to predict hydrodynamic load or hydrodynamic coefficients, e.g. Zhang et al. (2013), Joung et al. (2014), Bettle et al. (2014), Kumar et al. (2015), Luo and Lyu (2015), expressing the need for the synergy between lumped and continuum mechanics approaches.

Although the usage of CFD analysis to the subsea vehicles problems grows steadily, there are little works in the literature concerning numerical analysis of underwater manipulators, and especially we have not found any papers relating to the effects of current disturbances on the moments experienced by the manipulator joints. The present work was prepared with the intention to fill the gap in this area and to give a deeper insight into physics of the environmental disturbances caused by water current. Numerical simulations, performed by the use of ANSYS Fluent software, enabled us to observe the effects of such disturbances as changes in the current velocity direction and its magnitude on the moments experienced by the manipulator joints. The investigations of the current direction effect on the load on the manipulator were carried out as steady-state whilst those for the time dependent current speed, were considered as transient problem.

As a result of investigations the joint moments of hydrodynamic forces were plotted as a function of relative angle between the manipulator and the current and analyzed, showing that even small dissymmetry of the structure can cause unanticipated increase of the joint torque required to maintain the position of the arm. The time history of joint torques due to periodically varying with time current speed reveals that load variations follow gradually the variation of current speed for lower speed frequency, but 
when the period of change becomes short enough, the hydrodynamic load may change very sharply and violently due to the sudden pressure variations associated with changes of velocity field. These flow phenomena, which may strongly affect the underwater manipulators control, can be predicted quantitatively through appropriate experiments, still - CFD analysis may be considered as a useful and less expensive research tool in this regard. The present study is just a first step in modeling of hydrodynamic load on the underwater arm. The next step will focus on the problem of making the manipulator move through the application of dynamic meshes.

\section{REFERENCES}

1. ANSYS Inc. (2015), ANSYS FLUENT Theory Guide, Release 16.0, Canonsburg, USA.

2. Antonelli G. (2006), Underwater Robots (Springer Tracts in Advanced Robotics), Second edition, Springer.

3. Bettle M.C., Gerber A.G., Watt G.D. (2014), Using reduced hydrodynamic models to accelerate the predictor-corrector convergence of implicit 6-DOF URANS submarine manoeuvring simulations, Computers \& Fluids, 102, 215-236.

4. Fossen T.I. (1994), Guidance and Control of Ocean Vehicles, John Wiley \& Sons, Chichester, United Kingdom.

5. Herman P. (2009), Decoupled PD set-point controller for underwater vehicles, Ocean Engineering, 36, 529-534.

6. Joung T.-H., Choi H.-S., Jung S.-K., Sammut K., He F. (2014), Verification of CFD analysis method for predicting the drag force and thrust power of an underwater disc robot, International Journal of Naval Architecture and Ocean Engineering, 6, 269-281

7. Kumar M.S., Raja S.C., Kumar M.N.S, Gowthamraj B. (2015), A synergic approach to the conceptual design of Autonomous Underwater Vehicle, Robotics and Autonomous Systems, 67, 105-114.

8. Leabourne K.N., Rock S.M. (1998), Model Development of an Underwater Manipulator for Coordinated Arm-Vehicle Control, Proceedings of the OCEANS 98 Conference, Nice, France, 2, 941-946.
9. Luo W., Lyu W. (2015), An application of multidisciplinary design optimization to the hydrodynamic performances of underwater robots, Ocean Engineering, 104, 686-697.

10. McLain T.W., Rock S.M. (1998), Development and Experimental Validation of an Underwater Manipulator Hydrodynamic Model, The International Journal of Robotics Research, 17, 748-759.

11. Pazmino R. S., Garcia Cena C.E., Alvarez Arocha C., Santoja R.A. (2011), Experiences and results from designing and developing a $6 \mathrm{DoF}$ underwater parallel robot, Robotics and Autonomous System, 59, 101-112.

12. Richard M.J., Levesque B. (1996), Stochastic dynamic modelling of an open-chain manipulator in a fluid environment, Mechanism and Machine Theory, 31(5), 561-572.

13. Santhakumar M., Kim J. (2012), Indirect adaptive control of an autonomous underwater vehicle-manipulator system for underwater manipulation tasks, Ocean Engineering, 54, 233-243.

14. Vossoughi G.R., Meghdari A., Borhan H. (2004), Dynamic modeling and robust control of an underwater ROV equipped with a robotic manipulator arm, Proceedings of 2004 JUSFA, @004 Japan-USA Symposium on Flexible Automation, Denver, Colorado.

15. Wang Z. (2012), An interactive virtual prototyping platform considering environment effect described by fluid dynamics, Robotics and Computer-Integrated Manufacturing, 28, 316-325.

16. Zhang S., Yu J., Zhang A., Zhang F. (2013), Spiraling motion of underwater gliders: Modeling, analysis, and experimental results, Ocean Engineering, 60, 1-13.

This work has been supported by the Bialystok University of Technology grant No. S/WM/1/2012. 